\title{
The Rise of Stem Cell Toxicology
}

\author{
Francesco Faiola,* Nuoya Yin, Xinglei Yao, and Guibin Jiang
}

State Key Laboratory of Chemistry and Ecotoxicology, Research Center for Eco-Environmental Sciences, Chinese Academy of Sciences, Beijing 100085, China

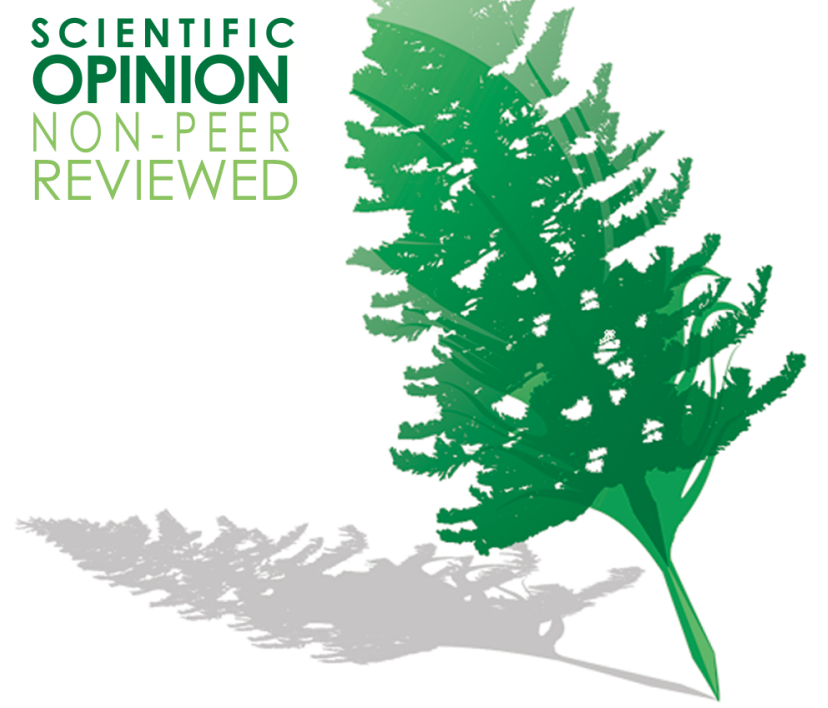

$\mathrm{T}$

he field of environmental toxicology has been challenged in the last few decades by the exponential discovery of novel persistent pollutants and the relative lack of knowledge of their hazardousness to human health. At the same time, there has been increasing awareness of the urgency and necessity of innovative, validated, and comprehensive assays to collect toxicity data more relevant to humans. Since the onset of toxicology science, we have heavily relied on animal tests. Although these in vivo experiments have been refined in recent years, they are still expensive, labor intensive, time-consuming, and accompanied by ethical issues. More importantly, Russell and Burch's "high fidelity fallacy" theory postulates that toxicity assays with animals are not always applicable to human health due to interspecies variations, even when primates are used. A prototypical example is the case of the drug thalidomide, proven to be highly teratogenic in humans even though it had passed all animal tests. ${ }^{1}$ This led to the introduction of in vitro tests as alternatives to in vivo animal experiments (reviewed in ref 2). In particular, the culture of human cells for toxicity tests, more directly relevant to human health, has been the primary solution to the obvious fact that humans cannot be used for in vivo studies. However, the direct derivation of some types of primary human cells, can be extremely invasive or simply impossible, and even if successful, the ability of primary cells to be cultured and expanded in vitro is limited. Conversely, immortalized or cancer cell lines can be readily grown and amplified in dishes; nevertheless, they may no longer be representative of the cells of origin because of accumulating mutations or altered cell functions, for example. Collectively, these issues can greatly affect the generation and interpretation of toxicity data. However, as described below, new stem cell technologies for the in vitro analyses of pollutants' potential hazardousness, allow scientists to move past these problems.

The first successful derivation of human embryonic stem cells (hESCs), ${ }^{3}$ and the discovery of human induced pluripotent stem cells (iPSCs), ${ }^{4}$ have intrigued the scientific community because of their potential to immensely advance many research fields. In particular, these breakthroughs are revolutionizing the field of toxicology in such a way that we need to create a new branch defined as "stem cell toxicology" that encompasses the thorough investigation of the adverse effects of substances on living organisms exclusively using stem cells. Though this approach may seem limited, it is important to remember that pluripotent stem cells have the capacity to proliferate indefinitely in culture, and the ability to differentiate into all cell types of an adult organism. These properties therefore make them ideal for human toxicological studies on various cell or tissue types. For instance, ESCs and iPSCs can be used to evaluate acute toxicity, and although other cultured cells types may be suitable for this type of assay, pluripotent stem cells have often been demonstrated to be more sensitive than somatic cells. The effects of a test material on fetal development can also be assessed with stem cells by looking at any abnormal differentiation phenotype. For example, ESCs can differentiate in vitro as three-dimensional aggregates called embryoid bodies (EBs), which mimic early embryonic developmental stages in vivo. This is the so-called embryonic/developmental toxicity assay. A third type of toxicity assay where stem cells are very useful is the cell function assay. Pluripotent stem cells are first differentiated into adult stem cells or progenitor cells, and then into particular types of terminally differentiated somatic cells. Subsequently, their cellular functions are assessed upon treatment with a chemical. This is particularly important when the primary cells are very difficult to derive directly from animals or humans. All of these assays are reviewed in. ${ }^{5} \mathrm{~A}$ growing concern about pollution is its relationship to human reproduction. With pluripotent stem cells, we are able to investigate in vitro the potential effects of pollutants on our ability to reproduce. In fact, murine ESCs can first be differentiated toward epiblast stem cells (EpiSCs), and then to germ cells. Similarly, hESCs can be differentiated toward primordial germ cell-like cells. We can then test whether these germ cells function correctly under various conditions.

In conclusion, stem cell toxicology allows for the concurrent assessment of many forms of toxicity including acute, embryonic, developmental, organ, reproductive, and functional. It also provides a unique and widely applicable system for

Received: March 27, 2015

Published: May 5, 2015 

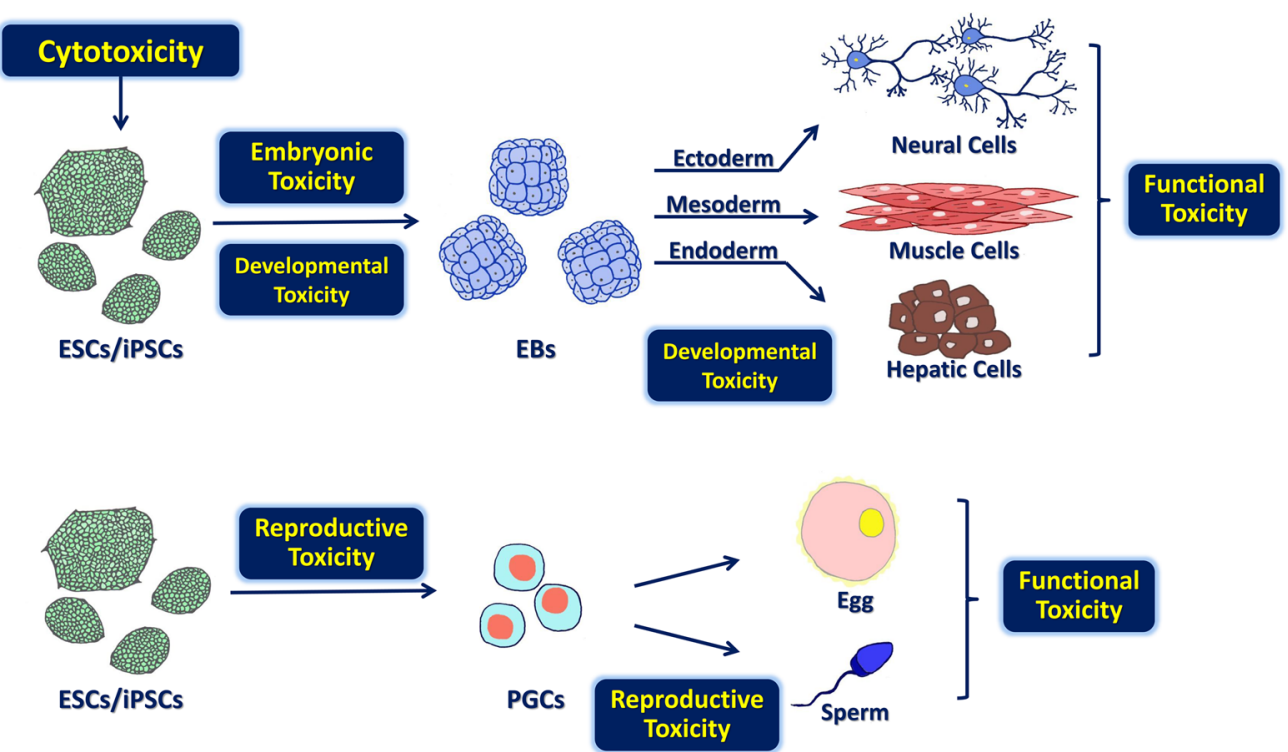

Figure 1. Stem cell-based toxicity assays. ESCs: Embryonic stem cells. iPSCs: Induced pluripotent stem cells. EBs: Embryoid bodies. PGCs: Primordial germ cells.

studies that are directly relevant to human health without the use of animal models (Figure 1). Although we have been stressing the importance of using human pluripotent stem cells in toxicology, we should also rely on murine ESCs and iPSCs, as well as human and mouse adult stem cells, to take advantage of all the scientific and technical advancements already achieved with them that we have not yet accomplished with the human and pluripotent counterparts. Indeed, the very first validated stem cell toxicity assay, the embryonic stem cell test (EST), was designed with murine cells by the European Center for the Validation of Alternative Methods (ECVAM) in 2002 (reviewed in ref 5). This simple test measured cell viability and the propensity to differentiate into clusters of beating cardiomyocytes; however, it was based exclusively on mouse ESCs using a single differentiation procedure, and without molecular end points to interpret toxicity. Thus, to appropriately define stem cell toxicology, we must include human stem cells to broaden the evaluation of toxicity. Nevertheless, before stem cell toxicology allows us to reach the goal of animal test-free toxicology, we need to not only perfect the myriad of traditional and novel stem cell differentiation procedures, but also to establish guidelines for stem cell-based toxicity assays. These should include universal procedures with validated molecular end points, which will greatly enhance the interpretation of toxicity data. Importantly, iPSC technology will enable us to assess toxicity without the ethical issues associated with the derivation and use of hESCs, but with the added benefit of the potential for personalized toxicology.

\section{AUTHOR INFORMATION}

\section{Corresponding Author}

*E-mail: faiola@rcees.ac.cn.

\section{Notes}

The authors declare no competing financial interest.

\section{REFERENCES}

(1) Mellin, G. W.; Katzenstein, M. The saga of thalidomide. Neuropathy to embryopathy, with case reports of congenital anomalies. N. Engl. J. Med. 1962, 267, 1184-92.

(2) Jennings, P. The future of in vitro toxicology. Toxicol. In Vitro 2014, DOI: $10.1016 /$ j.tiv.2014.08.011.

(3) Thomson, J. A.; Itskovitz-Eldor, J.; Shapiro, S. S.; Waknitz, M. A.; Swiergiel, J. J.; Marshall, V. S.; Jones, J. M. Embryonic stem cell lines derived from human blastocysts. Science 1998, 282 (5391), 1145-7.

(4) Takahashi, K.; Tanabe, K.; Ohnuki, M.; Narita, M.; Ichisaka, T.; Tomoda, K.; Yamanaka, S. Induction of pluripotent stem cells from adult human fibroblasts by defined factors. Cell 2007, 131 (5), 86172.

(5) Mori, H.; Hara, M. Cultured stem cells as tools for toxicological assays. J. Biosci. Bioeng. 2013, 116 (6), 647-52. 